\title{
EDITORIAL
}

\section{Cannabidiol in Parkinson's disease}

\author{
Carlos R. Rieder iD \\ Universidade Federal de Ciências da Saúde de Porto Alegre (UFCSPA), Porto Alegre, RS, Brazil, Irmandade da Santa Casa de Misericórdia \\ de Porto Alegre (ISCMPA), Porto Alegre, RS, Brazil.
}

Parkinson's disease (PD) is a common and complex neurological disorder that encompasses a range of clinical, epidemiological, and genetic subtypes. Loss of dopaminergic neurons in the substantia nigra leading to striatal dopamine depletion is the core mechanism underlying the cardinal motor features of PD. Although depletion of dopamine is the most notable neurotransmitter change in PD, other neurochemical changes occur and contribute to PD symptomatology. Many regions of the nervous system outside the basal ganglia are also involved in PD. The underlying molecular pathogenesis involves multiple pathways and mechanisms, such as $\alpha$-synuclein proteostasis, mitochondrial function, oxidative stress, calcium homeostasis, axonal transport, and neuroinflammation.

Although clinical diagnosis relies on the presence of cardinal motor features, PD is also associated with numerous non-motor symptoms that can be equally disabling than the motor symptoms or even more so. Drugs that enhance intracerebral dopamine concentrations or stimulate dopamine receptors remain the main treatment for motor symptoms. None of available treatments have proven to be neuroprotective or disease-modifying. Dopaminergic drugs are particularly effective during the early stages of the disease. However, PD invariably progresses, and long-term use of these medications may lead to reduced drug efficacy and the development of complications such as motor fluctuations and dyskinesias.

Unlike most of the motor features of PD, many nonmotor symptoms do not respond to dopaminergic therapy, and some are indeed aggravated by them, with great impact on patient quality of life. The refractoriness of these symptoms to dopaminergic therapy implicates nondopaminergic mechanisms. Therefore, current needs in the management of symptomatic patients with PD include dopamine-unresponsive axial motor impairments and non-motor symptoms, such as dementia, depression, anxiety, psychosis, and pain.

There is a clear need for therapies that target other pharmacological systems. A multimodal approach combining activity on dopaminergic as well as non-dopaminergic system would be very helpful and needs to be explored.

There has been interest in cannabidiol (CBD) as a treatment option for PD because of the identification of multiple potential targets of action in the CNS. CBD is one

Correspondence: Carlos R. Rieder

E-mail: carlosrieder@gmail.com

Submitted Dec 02 2019, accepted Dec 102019. of the many cannabinoids identified in Cannabis sativa, being the second most abundant constituent after $\triangle 9$-tetrahydrocannabinol (THC). Unlike THC, CBD is nonpsychoactive, and has been ascribed many potential medical benefits.

In this issue of the Brazilian Journal of Psychiatry, a research group from Faculdade de Medicina de Ribeirão Preto, Universidade de São Paulo, Brazil, addressed animal and human clinical studies involving the use of CBD for PD. ${ }^{1}$ The authors discussed the biological bases for a potential effect of CBD in this setting, as well as preclinical and clinical studies of CBD in PD. The latter, all conducted by their group, are an open-label study, ${ }^{2}$ a case series, ${ }^{3}$ and a randomized controlled trial. ${ }^{4}$ The open-label pilot study was conducted with six PD patients with psychotic symptoms, lasting at least 3 months before study entry, that could not be controlled by reduction of antiparkinsonian medications. ${ }^{2}$ Oral CBD doses ranging from 150-400 mg/day, combined with classic antiparkinsonian agents, reduced psychotic symptoms evaluated by different scales (the Brief Psychiatric Rating Scale and the Parkinson Psychosis Questionnaire), with no influence on cognitive and motor signs and no severe side effects. The second study was a case series of four PD patients with REM sleep behavior disorder (RBD). ${ }^{3}$ All had prompt, substantial, and persistent reductions in he frequency of RBD after CBD treatment. After drug discontinuation, the complex movements of $\mathrm{RBD}$ returned with baseline frequency and intensity. The third study was an exploratory double-blind trial of CBD versus placebo. ${ }^{4}$ Twenty-one PD patients without dementia or comorbid psychiatric conditions were assigned to three groups of seven subjects each who were treated with placebo, CBD $75 \mathrm{mg} /$ day, or CBD $300 \mathrm{mg} /$ day. Participants were assessed with respect to motor and general symptoms score (Unified Parkinson's Disease Rating Scale [UPDRS]) and wellbeing and quality of life (Parkinson's Disease Questionnaire [PDQ-39]). There were no differences across groups in motor score. However, the groups treated with CBD $300 \mathrm{mg} /$ day had significantly different mean total scores in the PDQ-39. The authors point to a possible effect of CBD in improving measures related to quality of life in PD patients without psychiatric comorbidities.

All of these studies showed interesting results, but sample sizes were very small and the duration of follow-up

How to cite this article: Rieder CR. Cannabidiol in Parkinson's disease. Braz J Psychiatry. 2020;42:126-127. http://dx.doi.org/10.1590/ 1516-4446-2019-0810 
was very short. The Movement Disorder Society EvidenceBased Medicine Committee recommendations for treatments of PD published in 2018 concluded that there was insufficient evidence to support the use of CBD for the treatment of PD at the time. ${ }^{5}$

It is vital to note that no conclusions can be drawn on the efficacy of CBD in this setting, as larger phase III and conclusive efficacy trials have not been conducted in PD. Double-blind, placebo-controlled, randomized trials with larger samples of patients with PD are needed to elucidate the possible effectiveness and mechanisms involved in the therapeutic potential of CBD in PD. Additionally, studies conducted specifically to evaluate the safety profile of CBD in patients with PD (including long-term safety), possible interactions with antiparkinsonian drugs, and possible side effects, as well as the therapeutic window for motor and non-motor PD symptoms, are also required.

\section{Disclosure}

CRR has been on the speakers' bureau/advisory board and/or has acted as a consultant for UCB
Biopharma S.A., Teva Farmacêutica, Roche Farmacêutica, and Medtronic.

\section{References}

1 Ferreira-Junior NC, Campos AC, Guimarães FS, Del-Bel E, Zimmermann PMR, Brum Junior L, et al. Biological bases for a possible effect of cannabidiol in Parkinson's disease. Braz J Psychiatry. 2020;42:218-24.

2 Zuardi AW, Crippa JA, Hallak JE, Pinto JP, Chagas MH, Rodrigues GG, et al. Cannabidiol for the treatment of psychosis in Parkinson's disease. J Psychopharmacol. 2009;23:979-83.

3 Chagas MH, Eckeli AL, Zuardi AW, Pena-Pereira MA, Sobreira-Neto MA, Sobreira ET, et al. Cannabidiol can improve complex sleeprelated behaviours associated with rapid eye movement sleep behaviour disorder in Parkinson's disease patients: a case series. J Clin Pharm Ther. 2014;39:564-6.

4 Chagas MH, Zuardi AW, Tumas V, Pena-Pereira MA, Sobreira ET, Bergamaschi MM, et al. Effects of cannabidiol in the treatment of patients with Parkinson's disease: an exploratory double-blind trial. J Psychopharmacol. 2014;28:1088-98.

5 Fox SH, Katzenschlager R, Lim SY, Barton B, de Bie RM, Seppi K, et al. International Parkinson and movement disorder society evidence-based medicine review: update on treatments for the motor symptoms of Parkinson's disease. Mov Disord. 2018;33:1248-66. 